\title{
A novel one-domain approach for modeling flow in a fluid-porous system including inertia and slip effects
}

\author{
Phys. Fluids 33, 022106 (2021); doi: 10.1063/5.0036812
}

F. J. Valdés-Parada ${ }^{\top}$ (D) and D. Lasseux ${ }^{2, a)}$ (D)

\begin{abstract}
AFFILIATIONS 09340 Ciudad de México, Mexico

2212M, UMR 5295, CNRS, Univ. Bordeaux, 351 Cours de la Libération, 33405 Talence Cedex, France

a) Author to whom correspondence should be addressed: didier.lasseux@u-bordeaux.fr
\end{abstract}

TDepartamento de Ingeniería de Procesos e Hidráulica, Universidad Autónoma Metropolitana-Iztapalapa, Av. San Rafael Atlixco 186,

\begin{abstract}
A new one-domain approach is developed in this work yielding an operational average description of one-phase flow in the classical Beavers and Joseph configuration including a porous medium topped by a fluid channel. The model is derived by considering three distinct regions: the homogeneous part of the porous domain, the inter-region, and the free fluid region. The development is carried out including inertial flow and slip effects at the solid-fluid interfaces. Applying an averaging procedure to the pore-scale equations, a unified macroscopic momentum equation, applicable everywhere in the system and having a Darcy form, is derived. The position-dependent apparent permeability tensor in this model is predicted from the solution of two coupled closure problems in the inter-region and in the homogeneous part of the porous medium. The performance of the model is assessed through in silico validations in different flow situations showing excellent agreement between the average flow fields obtained from direct numerical simulations of the pore-scale equations in the entire system and the prediction of the one-domain approach. Furthermore, validation with experimental data is also presented for creeping flow under no-slip conditions. In addition to the fact that the model is general from the point of view of the flow situations it encompasses, it is also simple and novel, hence providing a practical and interesting alternative to models proposed so far using one- or two-domain approaches.
\end{abstract}

\section{INTRODUCTION}

Modeling one-phase flow in a coupled fluid-porous medium system has been the subject of active research since the pioneering work of Beavers and Joseph. ${ }^{1,2}$ Applications of such a configuration are numerous, ranging from hydrology to chemical engineering. The main difficulty lies in a physically relevant way of reconciling a macroscopic description of the flow with a Darcy equation (or its variants) applicable in the bulk of the porous medium to the Navier-Stokes equation in the bulk fluid. Indeed, such a description requires one to account for the rapid variation of topology (and of the flow) in the separation zone between the two media, as evidenced, for instance, in the experimental works of Goharzadeh et al., ${ }^{3}$ Morad and Khalili, ${ }^{4}$ or more recently, by Terzis et al. ${ }^{5}$

As a generic configuration envisaged by Beavers and Joseph, ${ }^{1}$ consider the system sketched in Fig. 1 consisting of a channel partially filled with a porous medium. Let a single incompressible and
Newtonian fluid (the $\beta$-phase) flow through and above the porous medium under steady conditions. This system may be decomposed into three distinct regions (see Fig. 1) as follows.

\section{A. The $\omega$-region}

This region corresponds to the homogeneous part of the porous medium. For simplicity in the analysis, the $\sigma$-phase, representing the solid phase constitutive of the porous matrix, is assumed to be rigid and homogeneous. Consequently, in this region, the porosity, the seepage velocity, and the permeability are position-independent quantities. Here, Darcy's law, or its modifications, is applicable as a result of upscaling the microscale equations by making use of an averaging domain representative of the porous structure and physical mechanisms at play. Typically, the averaging volume corresponds to one or more unit cell(s) if the structure is assimilated to be a periodic one. 


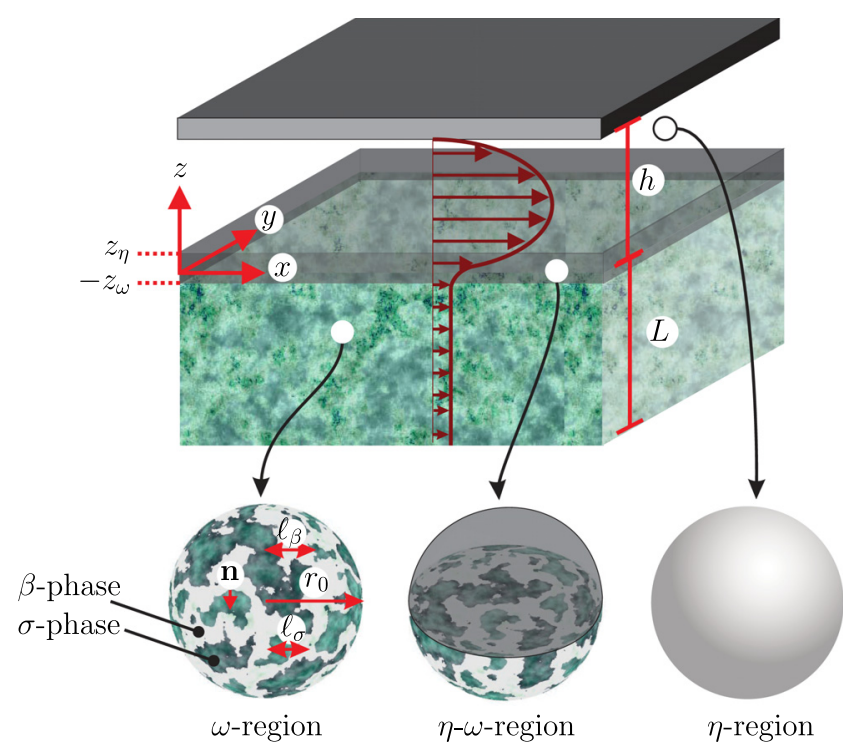

FIG. 1. Schematic representation of the flow in a channel partially obstructed with a porous medium, showing the three regions of the system, the volume averaging domain of radius $r_{0}$, and the characteristic lengths $L$ for the macroscopic size of the porous medium, $\ell_{\beta}$ in the fluid phase, $\beta$, and $\ell_{\sigma}$ in the solid phase, $\sigma$, within the porous medium. The $\omega, \eta-\omega$, and $\eta$ regions, respectively, correspond to $z \leq-z_{\omega},-z_{\omega} \leq z \leq z_{\eta}$, and $z_{\eta} \leq z \leq h$. The unit normal vector at the solidfluid interface, directed from the $\beta$-phase to the $\sigma$-phase, is denoted by $\mathbf{n}$.

\section{B. The $\eta-\omega$ inter-region}

This region is the transition zone where the average (or macroscopic) velocity experiences abrupt changes. For creeping-flow problems, the extent of this region has been proposed to be, at most, on the order of $10 \ell_{c},{ }^{6} \ell_{c}$ being the size of a geometrical unit cell representative of the porous material. Note that, even if the porous medium structure is assumed to be homogeneous, the velocity, the porosity, and the permeability exhibit spatial variations in this part of the system. Modeling flow in this region has been addressed using a generalized transport equation (or penalization approaches ${ }^{7,8}$ ) as explained below, or even by direct numerical simulations., ${ }^{9,10}$

\section{The $\eta$-region}

This region corresponds to the portion of the free fluid above the porous medium where the flow is one-dimensional and parallel to the channel axis. It is limited by the upper wall (also considered as the $\sigma$-phase) at $z=h$. Here, the volume fraction of the fluid phase in the averaging domain is equal to 1 , except near the walls, and flow is described by the Navier-Stokes equations.

Traditionally, flow and transport in this system have been studied using a two-domain approach, in which the average equations in the $\eta$-region are coupled to those in the $\omega$-region by means of suitable boundary conditions. Another alternative is the one-domain approach, in which a single average equation is used to describe transport in the three regions of the system. Both approaches have strengths and limitations, which are briefly discussed in the following paragraphs.

The two-domain approach has received far more attention than the one-domain approach. This may be explained by the fact that the model involves balance equations with constant transport coefficients, thus making their solution fairly easy to achieve, which represents an appealing strength. However, there is no consensus about which equations should be used in the porous medium and which boundary conditions should be employed. Originally, Beavers and Joseph ${ }^{1}$ proposed to couple Darcy's law with the Stokes equation by means of a jump condition in the velocity, which has the structure of a Newton's cooling law equation. Later, Neale and $\mathrm{Nader}^{11}$ proposed to couple the Darcy-Brinkman and Stokes equations with conditions of continuity of both the stress and velocity. Later, Ochoa-Tapia and Whitaker ${ }^{12}$ followed a similar path, albeit they proposed to consider a discontinuity in the viscous stress. The jump conditions proposed by both Beavers and Joseph ${ }^{1}$ and Ochoa-Tapia and Whitaker ${ }^{12}$ were written in terms of adjustable coefficients that needed to be determined experimentally. In addition, the position of the dividing surface where these boundary conditions are applied cannot be arbitrarily fixed. These issues have been discussed in many works. Among them is the one by ValdésParada et al., ${ }^{13}$ in which an iterative methodology was proposed to determine the dividing surface position as well as the values of the jump coefficients in these models. These authors also derived a more general two-domain model for which there are, in general, discontinuities of both the velocity and stress. Recently, the Beavers and Joseph boundary condition has been applied to inertial flow conditions, and it was found that the value of the adjustable coefficient increases with the Reynolds number. ${ }^{14}$ Nevertheless, this boundary condition has been recently found to be unsuitable for arbitrary flow directions. ${ }^{15}$ A more detailed review and discussion of the Beavers and Joseph boundary condition are available from Nield ${ }^{16}$ and Auriault. ${ }^{17}$

In the one-domain approach, the system is regarded as a pseudocontinuum, and a single equation is used to model the flow at the macroscopic scale everywhere. A heuristic or penalization approach is to postulate that such a model is the Navier-Stokes equation with a Darcy term involving a position-dependent permeability. A more rigorous approach was proposed by Ochoa-Tapia and Whitaker, ${ }^{12}$ who derived a generalized transport equation resulting from averaging and not upscaling the Stokes equation. This equation is more complicated than the penalization approach, and it has archival more than practical value. In fact, it was shown in this reference that the one-domain approach is necessary in the derivation of the jump conditions using the volume averaging method, ${ }^{18}$ justifying its archival value. Furthermore, the methodology proposed by Valdés-Parada et al. ${ }^{13}$ showed that it is necessary to account for the spatial variations of the effective coefficients present in the one-domain approach in order to determine the values of the jump coefficients and the dividing surface position. This difficulty in predicting the spatial variations of the permeability and porosity may explain why the one-domain approach has been less-frequently used than the two-domain approach. In addition, the corresponding average model is, in general, more complicated than the Darcy-Brinkman equation as originally derived by Ochoa-Tapia and Whitaker. ${ }^{12}$ The few applications of this modeling alternative use ad hoc coefficients and average expressions that are not always in agreement with the results obtained from pore-scale simulations (see for example Refs. 7,8 , and 19), and only an approximate closure scheme has been proposed to compute the spatial variations of the permeability. ${ }^{6}$ Moreover, existing analyses have been mainly focused on flow in the creeping regime, in the absence of rarefaction effects. 
The objective of this work is to address these issues by proposing a closed one-domain approach in the three-region system for one-phase flow including inertial and Knudsen effects in the slip regime. The model derived with this approach has a simple structure facilitating its application, nevertheless different from the modified Darcy-Brinkman equation used in the literature. The analysis is focused on the Beavers and Joseph configuration sketched in Fig. 1. The idea is to obtain average models that are valid in the three regions of the system and are coupled by continuity conditions using a simplified version of the volume averaging method. More specifically, this is achieved by deriving macroscale equations in both the $\omega$ - and $\eta-\omega$ regions in which the effective permeability tensors are obtained from coupled closure problems. The solution in the $\eta$-region is obtained analytically since the flow is one-dimensional along the channel axis $\left(\mathbf{e}_{x}\right)$ in this region. It is also coupled with that in the $\eta-\omega$ inter-region. The structure of the average models in the three regions is shown to involve a Darcylike momentum equation, which can be condensed into a single one-equation model with a position-dependent apparent permeability tensor. The solution of the closure scheme to obtain this tensor is computationally much less demanding than performing direct numerical simulations. The unified model is quite practical and appealing due to its simplicity. It is developed in a rather general flow context, in which inertial and/or slip effects at the solid/fluid interfaces may be present, widely extending the scope of the derived model with respect to the existing works reported so far. At this point, it is worth mentioning that there are some relevant works including slip effects in dual-porosity media; ${ }^{20-22}$ however, these works do not include inertial effects and do not account for the spatial variations of the apparent permeability tensor as it is done here.

With this aim in mind, the article is organized as follows. In Sec. II, the governing equations at the microscale are presented, and the derivations of the macroscale equations in each region are reported, yielding the unified one-domain momentum equation. Section III is dedicated to a series of illustrative examples showing the performance of the one-domain approach. This is achieved by comparisons of the flow fields obtained from direct numerical simulations at the microscale with predictions of the flow from the one-domain approach developed here. It is carried out for creeping flow in the absence and in the presence of slip effects as well as in the presence of inertia. In addition, validation is also presented by comparison with experimental data under creeping flow and no-slip conditions. Conclusions are drawn in Sec. IV.

\section{MICROSCALE MODEL AND DEVELOPMENT OF THE ONE-DOMAIN APPROACH}

The configuration under consideration is the one represented in Fig. 1. Before developing the one-domain macroscale model, the underlying boundary value flow problem must be formulated at the microscale. This is reported in Sec. II A followed by a rapid presentation of the derivation of the average mass-equation applicable everywhere in the system (Sec. II B). Finally, the macroscopic momentum transport equation is derived in each region (Secs. II C-II E).

\section{A. Microscale model}

The governing equations for mass and momentum transport at the pore-scale (in the three regions) are formulated in a rather general version in which inertia and/or slip effects may be present. Assuming incompressible flow, the mass and momentum balance equations in the $\kappa$-region $(\kappa=\omega, \eta \omega, \eta)$ are given by

$$
\begin{gathered}
\nabla \cdot \mathbf{v}_{\kappa}=0, \quad \text { in the } \beta \text {-phase, } \\
\rho \mathbf{v}_{\kappa} \cdot \nabla \mathbf{v}_{\kappa}=-\nabla p_{\kappa}+\mu \nabla^{2} \mathbf{v}_{\kappa}, \quad \text { in the } \beta \text {-phase. }
\end{gathered}
$$

In these two equations, $\rho$ and $\mu$ denote the fluid density and dynamic viscosity, both considered as constant, whereas $\mathbf{v}_{\kappa}$ and $p_{\kappa}$ represent the fluid velocity and pressure in the $\kappa$-region. Without loss of generality, volume forces are not considered in the momentum transport equation. Taking into account the possible existence of rarefaction effects, in particular if the flowing fluid is a gas, due to the size of the pores and/or channel in conjunction with the thermodynamic conditions, the interfacial boundary condition can be formulated as follows: ${ }^{23-26}$

$$
\mathbf{v}_{\kappa}=-\xi_{k} \lambda(\mathbf{I}-\mathbf{n n}) \cdot\left(\mathbf{n} \cdot\left(\nabla \mathbf{v}_{\kappa}+\nabla \mathbf{v}_{\kappa}^{T}\right)\right), \quad \text { at } \mathscr{A}_{\beta \sigma \kappa} .
$$

Here, I and $\mathbf{n}$, respectively, denote the identity tensor and the unit normal vector to the solid-fluid interface, $\mathscr{A}_{\beta \sigma \kappa}$, in the $\kappa$-region, directed from the $\beta$-phase to the $\sigma$-phase. In addition, $k=1$ for $\kappa=\omega, \eta \omega$ (i.e., at the solid-fluid interface inside the porous material) and $k=2$ when $\kappa=\eta$ (i.e., at the top surface of the channel). The mean free path of the fluid molecules is denoted by $\lambda$ while $\xi_{k}=\left(2-\sigma_{k}\right) / \sigma_{k}$ is a coefficient taking into account the reflection process of the molecules at the solid wall related to the tangential accommodation coefficient, $\sigma_{k}$. At this point, it is worth mentioning that the structure of the boundary condition given in Eq. (1c) is a Navier-type slip, and it is expressed in its complete form, i.e., including the transpose of the velocity gradient, which is different from previous works. ${ }^{27,28}$ Furthermore, this type of boundary condition has been proposed to study other physical mechanisms such as flow over rough surfaces. ${ }^{29,30}$ In this way, the above boundary condition is quite rich as it allows studying physical situations beyond only rarefaction effects. In addition, macroscopic boundary conditions at the inlet and outlet of the system (in the $x$-direction) should be provided to complete the statement of the pore-scale problem. However, they will not be used for the derivations that follow and are hence left unspecified here.

In order to carry out a macroscale description, an averaging domain, $\mathscr{V}$, of measure $V$ and characteristic size $r_{0}$, is defined. Two averaging operators are considered, namely, the superficial and intrinsic averages. For a piece-wise smooth function, $\psi$, defined in the fluid phase within the $\kappa$-region, they are, respectively, given by

$$
\begin{aligned}
\langle\psi\rangle_{\kappa} & =\frac{1}{V} \int_{\mathscr{V}_{\beta \kappa}} \psi d V, \\
\langle\psi\rangle_{\kappa}^{\beta} & =\frac{1}{V_{\beta \kappa}} \int_{\mathscr{V}_{\beta \kappa}} \psi d V,
\end{aligned}
$$

with $\kappa=\omega, \eta \omega, \eta$. In the above equations, $\mathscr{V}_{\beta \kappa}$ (of measure $V_{\beta \kappa}$ ) denotes the portion of the averaging domain occupied by the fluid phase in the $\kappa$-region. Both averaging operators are related according to

$$
\langle\psi\rangle_{\kappa}=\frac{V_{\beta \kappa}}{V}\langle\psi\rangle_{\kappa}^{\beta} .
$$

The ratio $V_{\beta \kappa} / V$ denotes the volume fraction of the fluid phase within the averaging domain and it is, in general, a function of position. In the porous medium bulk, this ratio is a constant corresponding to the porosity, $\varepsilon$. 


\section{B. Average mass balance equation}

The development starts with the derivation of the macroscopic mass balance equation in each region. Application of the superficial averaging operator to the continuity equation (1a) and use of the spatial averaging theorem ${ }^{31}$

$$
\langle\nabla \cdot \mathbf{a}\rangle_{\kappa}=\nabla \cdot\langle\mathbf{a}\rangle_{\kappa}+\frac{1}{V} \int_{\mathscr{A}_{\beta \sigma \kappa}} \mathbf{n} \cdot \mathbf{a} d A, \quad \kappa=\omega, \eta \omega, \eta
$$

(a denoting a vector field defined in the $\beta$-phase), together with the interfacial boundary condition [Eq. (1c)], leads to the macroscopic mass conservation equation which takes the same form in the threeregions. It is given by

$$
\nabla \cdot\left\langle\mathbf{v}_{\kappa}\right\rangle_{\kappa}=0, \quad \text { in the } \kappa-\text { region, } \kappa=\omega, \eta \omega, \eta .
$$

The attention must now be focused on the average momentum equation in each region and this is the purpose of Secs. II C-II E.

\section{Analysis in the homogeneous part of the porous medium ( $\omega$-region)}

This portion of the system has received considerable attention in the literature; hence, the derivations provided here are presented in a brief manner. More details can be found in references dedicated to the averaging of one-phase flow in a porous medium in the presence of inertia $^{32,33}$ and to the derivation of a macroscopic model for onephase flow when slip is present at the solid-fluid interfaces. ${ }^{26,34}$ For the sake of convenience, the structure of the porous medium is modeled as a periodic array of solid inclusions as illustrated in Fig. 2. Hence, the averaging domain, $\mathscr{V}$, may be defined as a finite array of unit cells (each of size $\ell_{c}$ ), with the following constraint for its characteristic size, $r_{0}$ :

$$
\ell \ll r_{0} \ll L
$$
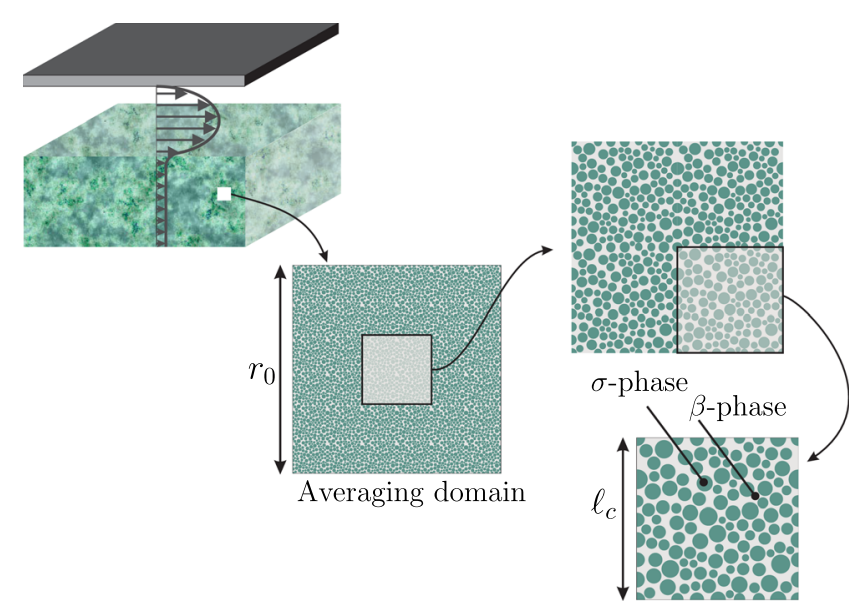

Unit cell

FIG. 2. Sketch of the averaging domain of size $r_{0}$ and a periodic two-dimensional unit cell (of size $\ell_{c}$ ) for the analysis of the flow problem in the homogeneous part of the porous medium. Note that, in general, $r_{0} \geq \ell_{c}$. The solid phase, $\sigma$, is represented as a random distribution of circular inclusions embedded in the fluid phase, $\beta$.
Here, $\ell$ represents the largest characteristic size at the pore-scale [i.e., $\left.\ell=\max \left(\ell_{\beta}, \ell_{\sigma}\right)\right]$, while $L$ is the smallest characteristic size of the porous medium at the macroscale (i.e., the smallest dimension of the porous medium in the three directions of space).

In order to derive the average momentum transport equation, it is convenient to follow the short-cut approach suggested by Barrère et al., ${ }^{35}$ in which Eqs. (1) are considered in the averaging domain, conceived as a Representative Elementary Volume (REV), made of one or more periodic unit cell(s) as sketched in Fig. 2. In this domain, it is reasonable to decompose the fluid pressure gradient according to ${ }^{36}$

$$
\nabla p_{\omega}=\nabla\left\langle p_{\omega}\right\rangle_{\omega}^{\beta}+\nabla \tilde{p}_{\omega},
$$

$\tilde{p}_{\omega}$ being the pressure deviations, and regard $\nabla\left\langle p_{\omega}\right\rangle_{\omega}^{\beta}$ as a constant within the REV. This assumption is justified on the basis of the separation of length scales given in (5). In this way, the pressure deviations and the fluid velocity can be assumed to be periodic at the inlets and outlets of the REV, and the pore-scale model can be written as follows:

$$
\begin{gathered}
\nabla \cdot \mathbf{v}_{\omega}=0, \quad \text { in } \mathscr{V}_{\beta \omega}, \\
\rho \mathbf{v}_{\omega} \cdot \nabla \mathbf{v}_{\omega}=-\nabla \tilde{p}_{\omega}+\mu \nabla^{2} \mathbf{v}_{\omega}-\nabla\left\langle p_{\omega}\right\rangle_{\omega}^{\beta}, \quad \text { in } \mathscr{V}_{\beta \omega}, \\
\mathbf{v}_{\omega}=-\xi_{1} \lambda(\mathbf{I}-\mathbf{n n}) \cdot\left(\mathbf{n} \cdot\left(\nabla \mathbf{v}_{\omega}+\nabla \mathbf{v}_{\omega}^{T}\right)\right), \quad \text { at } \mathscr{A}_{\beta \sigma \omega}, \\
\psi\left(\mathbf{r}+\mathbf{l}_{i}\right)=\psi(\mathbf{r}), \quad i=1,2,3 ; \psi=\mathbf{v}_{\omega}, \tilde{p}_{\omega}, \\
\left\langle\tilde{p}_{\omega}\right\rangle_{\omega}^{\beta}=0 .
\end{gathered}
$$

The last equation is an average constraint bounding the field of the pressure deviations that is compliant with the assumption that $\left\langle p_{\omega}\right\rangle_{\omega}^{\beta}$ is constant within the REV, and it is necessary for the flow problem to be well-posed. In Eq. (7d), $\mathbf{1}_{i}(i=1,2,3)$ represents the periodic lattice vectors of the REV.

The formal solution of this problem can be written as

$$
\begin{aligned}
& \mathbf{v}_{\omega}=-\frac{\mathbf{F}}{\mu} \cdot \nabla\left\langle p_{\omega}\right\rangle_{\omega}^{\beta}, \quad \text { in } \mathscr{V}_{\beta \omega}, \\
& \tilde{p}_{\omega}=-\mathbf{f} \cdot \nabla\left\langle p_{\omega}\right\rangle_{\omega}^{\beta}, \quad \text { in } \mathscr{V}_{\beta \omega},
\end{aligned}
$$

where $\mathbf{F}$ and $\mathbf{f}$ are closure variables that result from solving the following problem in the (periodic) REV

$$
\begin{gathered}
\nabla \cdot \mathbf{F}=\mathbf{0}, \quad \text { in } \mathscr{V}_{\beta \omega}, \\
-\frac{\rho}{\mu^{2}}\left\|\nabla\left\langle p_{\omega}\right\rangle_{\omega}^{\beta}\right\| \mathbf{e}_{p} \cdot \mathbf{F}^{T} \cdot \nabla \mathbf{F}=-\nabla \mathbf{f}+\nabla^{2} \mathbf{F}+\mathbf{I}, \quad \text { in } \mathscr{V}_{\beta \omega}, \\
\mathbf{F}=-\xi_{1} \lambda(\mathbf{I}-\mathbf{n n}) \cdot\left(\mathbf{n} \cdot\left(\nabla \mathbf{F}+(\nabla \mathbf{F})^{T 1}\right)\right), \quad \text { at } \mathscr{A}_{\beta \sigma \omega}, \\
\psi\left(\mathbf{r}+\mathbf{l}_{i}\right)=\psi(\mathbf{r}), \quad i=1,2,3 ; \psi=\mathbf{F}, \mathbf{f}, \\
\langle\mathbf{f}\rangle_{\omega}^{\beta}=\mathbf{0} .
\end{gathered}
$$

In Eq. (9b), $\mathbf{e}_{p}$ is a unit vector in the direction of the macroscopic pressure gradient. It is defined as

$$
\mathbf{e}_{p}=\frac{\nabla\left\langle p_{\omega}\right\rangle_{\omega}^{\beta}}{\left\|\nabla\left\langle p_{\omega}\right\rangle_{\omega}^{\beta}\right\|} .
$$

In addition, in Eq. (9c), the superscript $T 1$ denotes the transpose operator that permutes the first and second indices of a third order tensor $\mathbf{T}$, namely, $\mathbf{T}_{i j k}^{T 1}=\mathbf{T}_{j i k}$. 
Restricting the analysis to situations in which the macroscopic pressure gradient is $z$-independent, it follows that the same pressure gradient is experienced in the three regions of the system and therefore the subscript referring to the region for this term is dropped in the remainder of the analysis. Taking this into account, and applying the superficial averaging operator to Eq. (8a), yields

$$
\left\langle\mathbf{v}_{\omega}\right\rangle_{\omega}=-\frac{\mathbf{H}_{\omega}}{\mu} \cdot \nabla\langle p\rangle^{\beta}, \quad \text { in the } \omega \text {-region. }
$$

Here, $\mathbf{H}_{\omega}$ is the apparent permeability tensor given by

$$
\mathbf{H}_{\omega}=\langle\mathbf{F}\rangle_{\omega} .
$$

In summary, the macroscopic model in this region is given by Eqs. (4) and (11), which are in agreement with previous studies by Whitaker ${ }^{32}$ and Lasseux et al. ${ }^{33,34}$ among many others. In fact, when slip effects can be neglected, the corresponding closure problem was derived by Whitaker $^{32}$ and solved by Lasseux et al. ${ }^{33}$ These authors solved the closure problem given in Eq. (9) in a variety of flow situations. The derivation of the macroscale model in the presence of slip and without inertia in the homogeneous porous medium was also reported earlier. ${ }^{26,34}$ The symmetry properties of $\mathbf{H}_{\omega}$ were investigated by Lasseux and Valdés-Parada. ${ }^{37}$ Note that, for the particular problem envisaged by Beavers and Joseph, ${ }^{1}$ there are no inertial nor slip effects and the tensor $\mathbf{H}_{\omega}$ is the intrinsic permeability tensor. It must be noted that, for the sake of generality, the derivations are carried out considering that the flow in the $\omega$-region (and possibly the $\eta \omega$-region) may be multi-dimensional. Note also that the closure problem given in Eq. (9) is completely decoupled from the other regions of the system. For this reason, in the following, $\mathbf{F}$ is regarded as a given field.

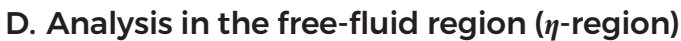

This portion of the system is located between $z=z_{\eta}$ and $z=h$ (see Fig. 1), the former corresponding to the position where the flow streamlines are horizontal. The criterion to define the location of $z_{\eta}$ will be provided later on. Consequently, $v_{\eta x} \equiv v_{\eta}$ is the only non-zero component of the velocity vector in this region so that the problem is defined by the following ordinary differential equation:

$$
0=\frac{d^{2} v_{\eta}}{d z^{2}}-\frac{1}{\mu} \frac{\partial\langle p\rangle^{\beta}}{\partial x}
$$

This equation is subject to the boundary conditions at $z=z_{\eta}$ and $z=h$. At the latter, the slip boundary condition can be written as

$$
v_{\eta}=-\xi_{2} \lambda \frac{d v_{\eta}}{d z}, \quad \text { at } z=h
$$

At $z=z_{\eta}$, it suffices, for the moment, to specify that this is a fictitious boundary at which it is physically justified to impose velocity and pressure continuity conditions. Combining the two at this stage of the development, allows to write

$$
\frac{d v_{\eta}}{d z}=\frac{d v_{\eta \omega x}}{d z}, \quad \text { at } z=z_{\eta} .
$$

In the above equation, $v_{\eta \omega x}$ is the $x$-component of the microscale velocity vector in the inter-region, which will be determined later. At this boundary, it is independent of $x$. Performing a semi-definite integration of Eq. (13), from $z_{\eta}$ to $z$ and making use of the boundary condition in Eq. (15), yields

$$
\frac{d v_{\eta}}{d z}=\frac{1}{\mu} \frac{\partial\langle p\rangle^{\beta}}{\partial x}\left(z-z_{\eta}\right)+\left.\frac{d v_{\eta \omega x}}{d z}\right|_{z=z_{\eta}} .
$$

Another step of semi-definite integration from $h$ to $z$ leads to

$$
\begin{aligned}
v_{\eta}(z)= & -\frac{\left(h-z_{\eta}\right)^{2}}{2 \mu} \frac{\partial\langle p\rangle^{\beta}}{\partial x}\left[1-\left(\frac{z-z_{\eta}}{h-z_{\eta}}\right)^{2}\right] \\
& +\left.\frac{d v_{\eta \omega x}}{d z}\right|_{z=z_{\eta}}(z-h)+v_{\eta}(h)
\end{aligned}
$$

From Eqs. (14) and (16), the expression of $v_{\eta}(h)$ is obtained as

$$
\left.v_{\eta}(h)=-\xi_{2} \lambda \frac{h-z_{\eta}}{\mu} \frac{\partial\langle p\rangle^{\beta}}{\partial x}+\left.\frac{d v_{\eta \omega x}}{d z}\right|_{z=z_{\eta}}\right),
$$

and the velocity in the $\eta$ region can hence be written as

$$
\begin{aligned}
v_{\eta}(z)= & -\frac{\left(h-z_{\eta}\right)^{2}}{2 \mu} \frac{\partial\langle p\rangle^{\beta}}{\partial x}\left[1+2 \xi_{2} \frac{\lambda}{h-z_{\eta}}-\left(\frac{z-z_{\eta}}{h-z_{\eta}}\right)^{2}\right] \\
& +\left.\frac{d v_{\eta \omega x}}{d z}\right|_{z=z_{\eta}}\left(z-h-\xi_{2} \lambda\right), \quad z_{\eta} \leq z \leq h .
\end{aligned}
$$

Clearly, this expression is not completely closed because $v_{\eta \omega x}$ is not available at this point of the analysis. This issue is addressed in the following paragraphs.

To conclude this section, the average velocity must now be derived by applying the superficial averaging operator to Eq. (19). In this region, the averaging domain reduces to a segment of size $r_{0}$ centered at $z$ and the average is given by

$$
\left\langle v_{\eta}\right\rangle_{\eta}=\frac{1}{r_{0}} \int_{\zeta=z-r_{0} / 2}^{\zeta=z_{\text {top }}} v_{\eta} d \zeta, \quad \text { when } z_{\eta} \leq z \leq h+r_{0} / 2
$$

with

$$
z_{\text {top }}=\min \left(z+r_{0} / 2, h\right),
$$

allowing to limit to $h$ the upper bound of the integration interval when $z \geq h-r_{0} / 2$. This gives

$$
\begin{aligned}
\left\langle v_{\eta}\right\rangle_{\eta}= & -\frac{\left(h-z_{\eta}\right)^{2}}{2 r_{0} \mu} \frac{\partial\langle p\rangle^{\beta}}{\partial x}\left[\left(1+2 \xi_{2} \frac{\lambda}{h-z_{\eta}}\right)\left(z_{\text {top }}-z+\frac{r_{0}}{2}\right)\right. \\
& \left.-\frac{\left(z_{\text {top }}-z_{\eta}\right)^{3}-\left(z-r_{0} / 2-z_{\eta}\right)^{3}}{3\left(h-z_{\eta}\right)^{2}}\right] \\
& +\left.\frac{1}{2 r_{0}} \frac{d v_{\eta \omega x}}{d z}\right|_{z=z_{\eta}}\left[\left(z_{\text {top }}-h-\xi_{2} \lambda\right)^{2}-\left(z-\frac{r_{0}}{2}-h-\xi_{2} \lambda\right)^{2}\right], \\
& z_{\eta} \leq z \leq h+r_{0} / 2 .
\end{aligned}
$$

Note that $\left\langle v_{\eta}\right\rangle_{\eta}$ corresponds to $v_{\eta}$ for $z \in\left[z_{\eta}, h-r_{0} / 2\right]$. In the zone near the upper wall (i.e., for $z \in\left[h-r_{0} / 2, h+r_{0} / 2\right]$ ), the correspondence can only be achieved on average. 


\section{E. Analysis in the fluid-porous medium inter-region ( $\eta-\omega$ region)}

This transition zone is comprised between $z=-z_{\omega}$ (below which the homogeneous porous medium begins) and $z=z_{\eta}$ (above which the free fluid region commences) as sketched in Fig. 3. At this boundary, the condition of continuity of pressure has already been used. Hence the only remaining option is to impose that

$$
\mathbf{v}_{\eta \omega}=v_{\eta}\left(z_{\eta}\right) \mathbf{e}_{x}, \quad \text { at } z=z_{\eta},
$$

or, after substitution of Eq. (19),

$$
\begin{aligned}
\mathbf{v}_{\eta \omega}= & -\frac{\left(h-z_{\eta}\right)^{2}}{2 \mu}\left(1+2 \xi_{2} \frac{\lambda}{h-z_{\eta}}\right) \frac{\partial\langle p\rangle^{\beta}}{\partial x} \mathbf{e}_{x} \\
& +\left.\frac{\partial v_{\eta \omega x}}{\partial z}\right|_{z=z_{\eta}}\left(z_{\eta}-h-\xi_{2} \lambda\right) \mathbf{e}_{x}, \quad \text { at } z=z_{\eta} .
\end{aligned}
$$

This equation can be reformulated after using the identities $\frac{\partial\langle p\rangle^{\beta}}{\partial x} \mathbf{e}_{x}$ $=\nabla\langle p\rangle^{\beta}$ and $\left.\frac{\partial v_{\operatorname{\eta nx}}}{\partial z}\right|_{z=z_{\eta}} \mathbf{e}_{x} \equiv \mathbf{n} \cdot \nabla \mathbf{v}_{\eta \omega}$ to obtain the following expression:

$$
\begin{aligned}
\mathbf{v}_{\eta \omega}= & -\frac{\left(h-z_{\eta}\right)^{2}}{2 \mu}\left(1+2 \xi_{2} \frac{\lambda}{h-z_{\eta}}\right) \nabla\langle p\rangle^{\beta} \\
& +\left(z_{\eta}-h-\xi_{2} \lambda\right) \mathbf{n} \cdot \nabla \mathbf{v}_{\eta \omega}, \quad \text { at } z=z_{\eta} .
\end{aligned}
$$

In addition, the mass and momentum transport equations at the porescale in this region can be written as

$$
\begin{gathered}
\nabla \cdot \mathbf{v}_{\eta \omega}=0, \quad \text { in } \mathscr{V}_{\beta \eta \omega}, \\
\rho \mathbf{v}_{\eta \omega} \cdot \nabla \mathbf{v}_{\eta \omega}=-\nabla \tilde{p}_{\eta \omega}+\mu \nabla^{2} \mathbf{v}_{\eta \omega}-\nabla\langle p\rangle^{\beta}, \quad \text { in } \mathscr{V}_{\beta \eta \omega} .
\end{gathered}
$$

Moreover, at the solid-fluid interface, the slip condition is still applicable,

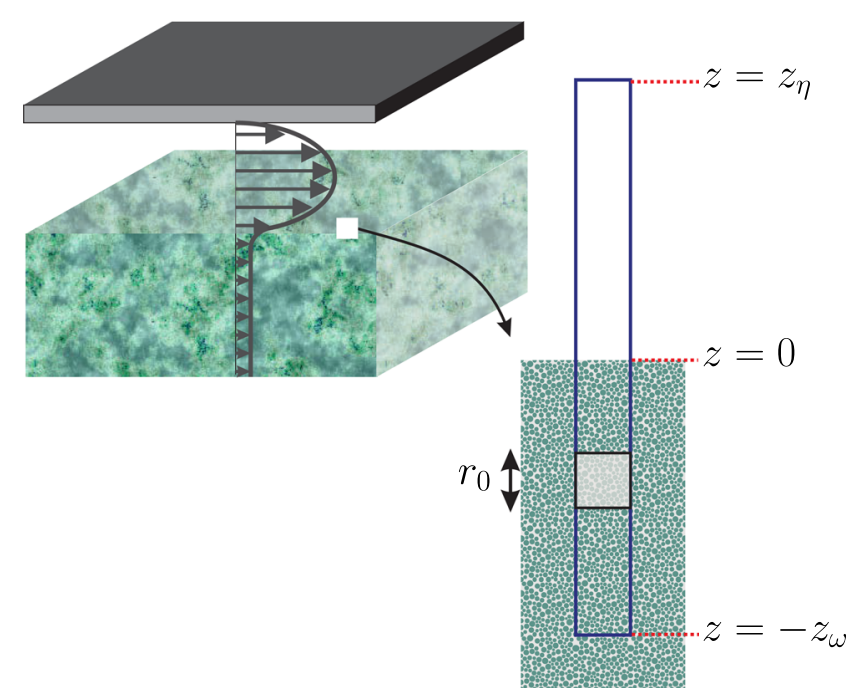

FIG. 3. Sketch of the domain used to study flow in the inter-region. The gray square represents the averaging domain (the REV) of size $r_{0}$, which can be located anywhere within this zone of the system.

$$
\mathbf{v}_{\eta \omega}=-\xi_{1} \lambda(\mathbf{I}-\mathbf{n n}) \cdot\left(\mathbf{n} \cdot\left(\nabla \mathbf{v}_{\eta \omega}+\nabla \mathbf{v}_{\eta \omega}^{T}\right)\right), \quad \text { at } \mathscr{A}_{\beta \sigma \eta \omega} .
$$

Furthermore, at the lower boundary of the inter-region, it is acceptable to impose the condition of continuity of the velocity fields, i.e.,

$$
\mathbf{v}_{\eta \omega}=\mathbf{v}_{\omega}=-\frac{\mathbf{F}}{\mu} \cdot \nabla\langle p\rangle^{\beta}, \quad \text { at } z=-z_{\omega} .
$$

These equations are to be solved in a vertical stripe of the REV that is periodic only in the two horizontal, $x$ and $y$, directions (see Fig. 1). Hence, it is reasonable to impose the following boundary condition at the lateral boundaries of the solution domain

$$
\psi\left(\mathbf{r}+\mathbf{l}_{i}\right)=\psi(\mathbf{r}), \quad i=1,2 ; \psi=\mathbf{v}_{\eta \omega}, \tilde{p}_{\eta \omega},
$$

where, again, $\mathbf{l}_{i}(i=1,2)$ denotes the periodic lattice vectors of the REV. Finally, the pressure deviations are bound by

$$
\left\langle\tilde{p}_{\eta \omega}\right\rangle^{\beta}=0 \text {. }
$$

The formal solution of this problem is given by

$$
\begin{aligned}
& \mathbf{v}_{\eta \omega}=-\frac{\mathbf{D}}{\mu} \cdot \nabla\langle p\rangle^{\beta}, \quad \text { in } \mathscr{V}_{\beta \eta \omega}, \\
& \tilde{p}_{\eta \omega}=-\mathbf{d} \cdot \nabla\langle p\rangle^{\beta}, \quad \text { in } \mathscr{V}_{\beta \eta \omega} .
\end{aligned}
$$

The closure variables $\mathbf{D}$ and $\mathbf{d}$ solve the following boundary-value problem:

$$
\begin{gathered}
\mathbf{D}=\frac{\left(h-z_{\eta}\right)^{2}}{2}\left(1+2 \xi_{2} \frac{\lambda}{h-z_{\eta}}\right) \mathbf{I} \\
+\left(z_{\eta}-h-\xi_{2} \lambda\right) \mathbf{n} \cdot \nabla \mathbf{D}, \quad \text { at } z=z_{\eta} \\
\nabla \cdot \mathbf{D}=\mathbf{0}, \quad \text { in } \mathscr{V}_{\beta \eta \omega}, \\
-\frac{\rho}{\mu^{2}}\left\|\nabla\langle p\rangle^{\beta}\right\| \mathbf{e}_{p} \cdot \mathbf{D}^{T} \cdot \nabla \mathbf{D}=-\nabla \mathbf{d}+\nabla^{2} \mathbf{D}+\mathbf{I}, \quad \text { in } \mathscr{V}_{\beta \eta \omega}, \\
\mathbf{D}=-\xi_{1} \lambda(\mathbf{I}-\mathbf{n n}) \cdot\left(\mathbf{n} \cdot\left(\nabla \mathbf{D}+(\nabla \mathbf{D})^{T 1}\right)\right), \quad \text { at } \mathscr{A}_{\beta \sigma \eta \omega}, \\
\mathbf{D}=\mathbf{F}, \quad \text { at } z=-z_{\omega}, \\
\psi\left(\mathbf{r}+\mathbf{l}_{i}\right)=\psi(\mathbf{r}), \quad i=1,2 ; \psi=\mathbf{D}, \mathbf{d} \\
\langle\mathbf{d}\rangle^{\beta}=\mathbf{0} .
\end{gathered}
$$

The average momentum transport equation results from applying the superficial averaging operator to Eq. (24a), yielding

$$
\left\langle\mathbf{v}_{\eta \omega}\right\rangle_{\eta \omega}=-\frac{\mathbf{H}_{\eta \omega}}{\mu} \cdot \nabla\langle p\rangle^{\beta}, \quad \text { in the } \eta \text { - } \omega \text { inter-region. }
$$

Here, the position-dependent apparent permeability tensor is defined as

$$
\mathbf{H}_{\eta \omega}=\langle\mathbf{D}\rangle_{\eta \omega} .
$$

Evidently, the size of the inter-region is larger than that of the averaging domain in this case. Consequently, once the closure problem is solved, providing the fields of $\mathbf{D}$ and $\mathbf{d}$ in the entire $\eta-\omega$ region, several averaging steps are needed by positioning the averaging domain between $z=-z_{\omega}$ and $z=z_{\eta}$ in order to describe the spatial variations of $\mathbf{H}_{\eta \omega}$.

Now that an expression for $\mathbf{v}_{\eta \omega}$ is available, it is possible to return the attention to Eq. (20c) and substitute the result given in Eq. (24a) to obtain 


$$
\begin{aligned}
\left\langle\mathbf{v}_{\eta}\right\rangle_{\eta}= & -\frac{1}{\mu}\left\{\frac { ( h - z _ { \eta } ) ^ { 2 } } { 2 r _ { 0 } } \left[\left(1+2 \xi_{2} \frac{\lambda}{h-z_{\eta}}\right)\left(z_{\text {top }}-z+\frac{r_{0}}{2}\right)\right.\right. \\
& \left.-\frac{\left(z_{\text {top }}-z_{\eta}\right)^{3}-\left(z-r_{0} / 2-z_{\eta}\right)^{3}}{3\left(h-z_{\eta}\right)^{2}}\right] \mathbf{I}+\frac{1}{2 r_{0}}\left[\left(z_{\text {top }}-h-\xi_{2} \lambda\right)^{2}\right. \\
& \left.\left.-\left(z-\frac{r_{0}}{2}-h-\xi_{2} \lambda\right)^{2}\right]\left.\mathbf{n} \cdot \nabla \mathbf{D}\right|_{z=z_{\eta}}\right\} \cdot \mathbf{e}_{x} \mathbf{e}_{x} \cdot \nabla\langle p\rangle^{\beta}
\end{aligned}
$$$$
\text { in the } \eta \text {-region. }
$$

To simplify this expression, one may define the second-order apparent permeability tensor

$$
\begin{aligned}
\mathbf{H}_{\eta}= & \left\{\frac { ( h - z _ { \eta } ) ^ { 2 } } { 2 r _ { 0 } } \left[\left(1+2 \xi_{2} \frac{\lambda}{h-z_{\eta}}\right)\left(z_{\text {top }}-z+\frac{r_{0}}{2}\right)\right.\right. \\
& \left.-\frac{\left(z_{\text {top }}-z_{\eta}\right)^{3}-\left(z-r_{0} / 2-z_{\eta}\right)^{3}}{3\left(h-z_{\eta}\right)^{2}}\right] \mathbf{I}+\frac{1}{2 r_{0}}\left[\left(z_{\text {top }}-h-\xi_{2} \lambda\right)^{2}\right. \\
& \left.\left.-\left(z-\frac{r_{0}}{2}-h-\xi_{2} \lambda\right)^{2}\right]\left.\mathbf{n} \cdot \nabla \mathbf{D}\right|_{z=z_{\eta}}\right\} \cdot \mathbf{e}_{x} \mathbf{e}_{x}
\end{aligned}
$$

In this way, the models in the three regions consist of the mass balance equation (4) and the following momentum equations:

$$
\begin{gathered}
\left\langle\mathbf{v}_{\eta}\right\rangle_{\eta}(z)=-\frac{\mathbf{H}_{\eta}(z)}{\mu} \cdot \nabla\langle p\rangle^{\beta}, \quad \text { in the } \eta \text {-region, } \\
\left\langle\mathbf{v}_{\eta \omega}\right\rangle_{\eta \omega}(z)=-\frac{\mathbf{H}_{\eta \omega}(z)}{\mu} \cdot \nabla\langle p\rangle^{\beta}, \quad \text { in the } \eta-\omega \text { inter-region, } \\
\left\langle\mathbf{v}_{\omega}\right\rangle_{\omega}=-\frac{\mathbf{H}_{\omega}}{\mu} \cdot \nabla\langle p\rangle^{\beta}, \quad \text { in the } \omega \text {-region. }
\end{gathered}
$$

Since these three equations have the same structure, it is convenient to propose the following one-domain approach expression:

$$
\langle\mathbf{v}\rangle(z)=-\frac{\mathbf{H}(z)}{\mu} \cdot \nabla\langle p\rangle^{\beta}, \quad \text { everywhere. }
$$

Here, the position-dependent apparent permeability tensor $\mathbf{H}$ is defined as

$$
\mathbf{H}(z)= \begin{cases}\mathbf{H}_{\eta}(z), & \text { in the } \eta \text {-region } \\ \mathbf{H}_{\eta \omega}(z), & \text { in the } \eta \text { - } \omega \text { inter-region } \\ \mathbf{H}_{\omega}, & \text { in the } \omega \text {-region }\end{cases}
$$

with the tensors $\mathbf{H}_{\eta}(z), \mathbf{H}_{\eta \omega}(z)$, and $\mathbf{H}_{\omega}$, respectively, given in Eqs. (29), (27), and (12). The model given in Eq. (31) is quite appealing due to its resemblance to Darcy's law.

At this point, it is pertinent to clarify how the inter-region bounds, namely, $-z_{\omega}$ and $z_{\eta}$, can be determined. The former is specified as follows: first a value of $-z_{\omega}$ is proposed and $\mathbf{H}_{\eta \omega}\left(-z_{\omega}+r_{0} / 2\right)$ is computed. Second, this prediction is compared with $\mathbf{H}_{\omega}$. If these values do not satisfy a given tolerance, then the initial guess for $-z_{\omega}$ is further decreased, and the process is repeated until convergence is reached. Regarding the value of $z_{\eta}$, an iterative process may also be employed. In this case, convergence is decided with respect to the dependence of the closure variable $\mathbf{D}$ with $x$ (in a two-dimensional system) within the REV. When the value of $z_{\eta}$ is such that the field of $\mathbf{D}$ is $x$-independent (to within a given tolerance), it is assumed that $z_{\eta}$ is adequate.

The solution of the closure problem in the inter-region is the step that is computationally the more demanding. However, the numerical requirements are considerably smaller than those needed for the solution of the pore-scale problem in the entire three-region domain. In addition, the derivation of the model did not require to adopt more assumptions than those involved in the statement of the governing equations at the pore-scale and the one expressed in (5) only in the $\omega$-region. Furthermore, the model that is formulated in this work is more general than the one proposed in previous works for the Beavers and Joseph problem as it encompasses inertial and slip effects and it does not make use of heuristic approximations. It is also considerably simpler and more practical. Its predictive capabilities are illustrated in the following paragraphs with respect to direct numerical simulations and experimental data.

\section{RESULTS}

The objective of this section is to present some numerical results on model structures in order to validate the model derived above and illustrate its performance. This is carried out in three distinct situations, first in the classical creeping regime without slip effects (Sec. III A), second in the presence of inertia (Sec. III B), and third in the creeping regime with slip effects (Sec. III C). The validation is carried out by performing first a direct numerical simulation (DNS) of the pore-scale problem by solving Eq. (1) over the entire macroscopic system. These numerical results are then averaged using an averaging volume of size $r_{0}$ and are compared to the predictions of the one-domain approach (ODA) developed in this work. To do so, this requires the solution of the two closure problems given in Eqs. (9) (for the $\omega$ region) and (25) (for the $\eta-\omega$ region), respectively. While the former is solved in a REV which reduces to a periodic unit cell of the porous structure, the latter is solved in the entire inter-region. Before solving the boundary-value problems for the closure and pore-scale physical variables, they were first formulated in terms of the following dimensionless variables and parameters:

$$
\begin{aligned}
& \mathbf{r}^{*}=\frac{\mathbf{r}}{\ell_{c}} ; \quad \mathbf{v}^{*}=\frac{\mathbf{v}}{v_{r e f}} ; \quad p^{*}=\frac{\ell_{c} p}{\mu v_{r e f}} ; \quad R e=\frac{\rho v_{r e f} \ell_{c}}{\mu} ; \\
& \lambda^{*}=\frac{\lambda}{\ell_{c}} ; \quad \mathbf{D}^{*}=\frac{\mathbf{D}}{\ell_{c}^{2}} ; \quad \mathbf{d}^{*}=\frac{\mathbf{d}}{\ell_{c}} ; \quad \mathbf{F}^{*}=\frac{\mathbf{F}}{\ell_{c}^{2}} ; \quad \mathbf{f}^{*}=\frac{\mathbf{f}}{\ell_{c}} .
\end{aligned}
$$

Here, the reference velocity is chosen to be $v_{\text {ref }}=\ell_{c}^{2}\left\|\nabla\langle p\rangle^{\beta}\right\| / \mu$. In this way, an alternative definition for the Reynolds number is $R e=\rho \ell_{c}^{3}|| \nabla\langle p\rangle^{\beta} \| / \mu^{2}$. In addition, $\xi_{k} \lambda^{*}$ represents the cell-Knudsen numbers in the porous medium $(k=1)$ and at the channel upper wall $(k=2)$. The dimensionless forms of the average model and the closure problems are not provided here for the sake of brevity in presentation. Nevertheless, it is worth mentioning that the Reynolds and cellKnudsen numbers account for the physical nature of the gas and are the only physical parameters of importance in the model.

The numerical solutions were carried out using the finite element software Comsol Multiphysics 5.5 for both the flow and closure problem solutions. A triangular unstructured mesh was employed, and the direct PARADISO solver was chosen. Mesh convergence tests were systematically performed by making use of an adaptive mesh 
refinement procedure and the minimum mesh size was selected when a tolerance of $10^{-4}$ for the maximum relative error between two successive refinements was reached. In this way, all the numerical results reported in this section can be considered independent of this numerical degree of freedom. In the following paragraphs, the numerical results are presented for specific flow conditions.

The numerical tests were performed considering two types of porous structures. The first one, referred to as "Type 1 " in the following, is made of a regular array of in-line parallel cylinders of circular cross section, arranged on a square pattern. The second one ("Type 2 ") is a periodic repetition of a unit cell, such as the one sketched in Figs. 2 and 3, composed of a random distribution in position and size of parallel cylinders of circular cross section. The numerical tests were carried out considering a constant macroscopic pressure gradient imposed along the channel axis, $\mathbf{e}_{x}$, orthogonal to the cylinder axes. Due to the chosen scaling, the dimensionless pressure gradient magnitude is 1 . The system is assumed to be of infinite extent in the $y$-direction.

At the pore-scale, the flow is two-dimensional in the $x z$-plane. For the DNS, the solution of the dimensionless version of Eq. (1) was hence computed in a domain that only includes one unit cell in the $x$-direction. Periodic conditions for $\tilde{p}_{\kappa}^{*}$ and $\mathbf{v}_{\kappa}^{*}(\kappa=\eta, \eta \omega, \omega)$ are imposed in this direction. The solution in the $\omega$-region was sought in only one unit cell in the $z$-direction below $z=-z_{\omega}$. Periodic boundary conditions for the velocity and pressure deviations were imposed in the $x$ - and $z$-directions between $z=-z_{\omega}$ and $z=-z_{\omega}-\ell_{c}$. In order for the problem to be well-posed, the intrinsic average of the pressure deviations over the entire computational domain was set to be zero.

At the macroscopic scale, both Type 1 and Type 2 porous structures are orthotropic in the $x z$ plane (i.e., $H_{\kappa x z}=H_{\kappa z x}=0$, $\kappa=\eta \omega, \omega)$ so that the average flow is along $\mathbf{e}_{x}$ in the three regions for both structures, requiring only the computation of $H_{k x x}$. To this end, the closure problems given in Eqs. (9) and (25) were numerically solved in a coupled manner in a single unit cell for the $\omega$-region and in the entire $\eta-\omega$ region, respectively. It was verified that taking $z_{\eta}=r_{0}$ and $z_{\omega}=11 \ell_{c}$ satisfied the above criteria for all the cases reported in the present section. In some cases, this size of the interregion turned out to be much larger than required; however, this does not constitute a limitation in the results. In other words, the interest was not focused on the precise determination of the inter-region width but in providing a solution domain for the closure problem that is large enough to carry out the analysis for all the situations envisaged here. Once the closure problems solutions were available, they were substituted into Eqs. (29), (27) and (12), to compute $H_{\eta x x}, H_{\eta \omega \mathrm{xx}}$ and $H_{\omega x x}$, respectively. With these results at hand, the ODA average velocity was computed using Eq. (31).

\section{A. Creeping flow regime}

The first test is carried out considering the simple situation in which the flow remains in the creeping regime under no-slip conditions (i.e., for $\xi_{1} \lambda=\xi_{2} \lambda=0$ ). In this case, the tensor $\mathbf{H}_{\omega}=H_{\omega x x} \mathbf{I}$ corresponds to the intrinsic permeability of the porous medium.

An example of the resulting velocity profile is reported in Fig. 4. In this case, the porous medium geometry is of Type 1 , the porosity was taken to be 0.8 and the channel height was fixed to be $h=100 \ell_{c}$. The value of the intrinsic permeability, obtained from Eq. (12) is

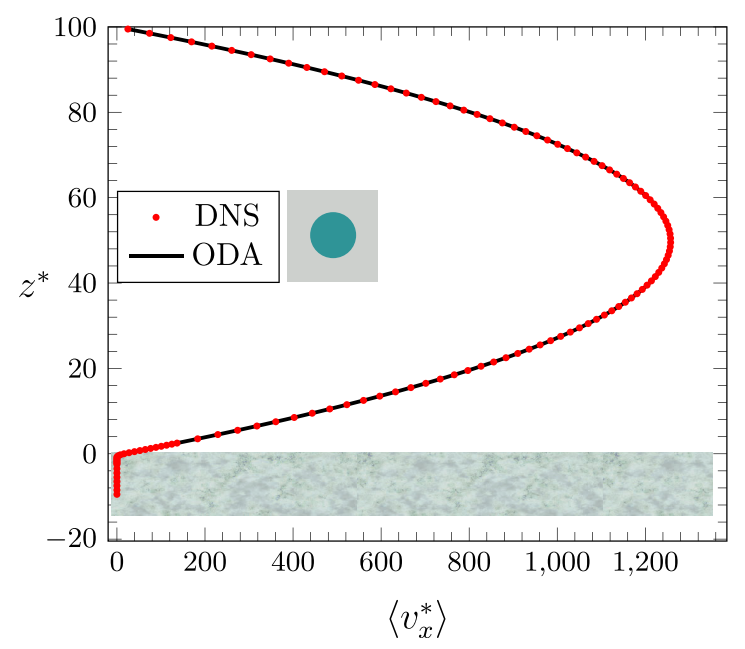

FIG. 4. Comparison of the velocity profiles predicted from the one-domain approach (ODA) and obtained from the averaged fields from direct-numerical simulations (DNS) in the entire system taking $r_{0}^{*}=1, \varepsilon=0.8$, and $h^{*}=100$.

$H_{\omega x x}=0.01947 \ell_{c}^{2}$, which is in perfect agreement with the estimate from the analytical expression provided by Chai et al. ${ }^{38}$ This represents a first validation of the numerical procedure employed here. In the same figure, the average velocity profile resulting from DNS is also reported. Both the DNS and ODA results were obtained by using an averaging domain size equal to that of the geometrical unit cell, i.e., $r_{0}^{*}=1$. Clearly, excellent agreement between both sets of results is achieved everywhere. In addition, it was verified that in the zone corresponding to $z \in\left[z_{\eta}, h-r_{0} / 2\right]$, the ODA results reproduced the values of $v_{\eta}$ obtained from the DNS. It should be noted that the average velocity at the top wall is not zero and this is due to the fact that at this position, there is still a portion of the averaging domain that includes fluid in the channel.

It is now of interest to investigate the influence of the porous medium geometry and of the averaging domain size, $r_{0}$, on the predictions of the average velocity in the inter-region. To carry this out, simulations were performed considering the porous structures of Type 1 and Type 2, different values of the porosity and values of $r_{0}^{*}$ ranging between 1 and 10. In specific, comparison of the velocity profiles in the $\eta$ - $\omega$ inter-region obtained from DNS and predicted from the ODA are reported in Figs. 5(a) and 5(b) for the porous structure of Type 1 taking $\varepsilon=0.8$ and $\varepsilon=0.4$, respectively. A similar comparison, considering the porous structure of Type 2 and $\varepsilon=0.4$, is represented in Fig. 5(c). Obviously, the porous medium microstructure plays a relevant role in the homogeneous part of the porous medium and in the inter-region, but the effect is lost for $z^{*}>5$ in the fluid phase. In particular, it is interesting to note the considerable contrast in the velocity profiles presented in Figs. 5(b) and 5(c), both corresponding to the same porosity but different distributions of the solid inclusions, i.e., ordered (Type 1) and disordered (Type 2) structures, respectively. For the porous structure of Type 2, $\left\langle v_{\omega x}\right\rangle_{\omega}$ decreases in about three orders of magnitude with respect to the values obtained with the porous structure of Type 1, as a direct result of the same decrease in $H_{\omega x x}$. Interestingly, the disordered structure seems to attenuate the oscillations in the velocity profiles in the inter-region, in particular 

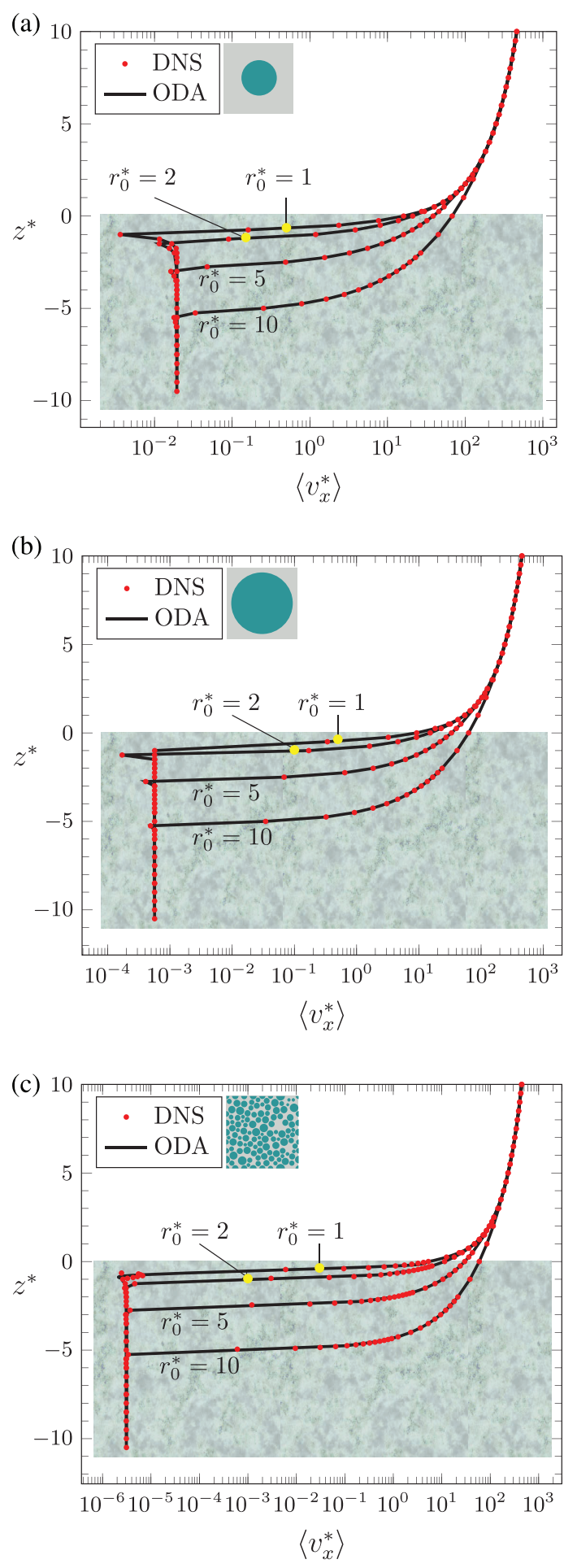

FIG. 5. Comparison of the average velocity profiles predicted from the one-domain approach (ODA) and the averaged velocity obtained from direct numerical simulations (DNS) near the fluid-porous medium boundary taking $h^{*}=100$ and four values of $r_{0}^{*}$ for (a) the porous structure of Type $1, \varepsilon=0.8$, (b) the porous structure of Type $1, \varepsilon=0.4$, and (c) the porous structure of Type 2, $\varepsilon=0.4$. close to the free fluid boundary (i.e., close to $z=0$ ). As shown in Fig. 5(a), these oscillations are quite noticeable as the porosity is increased. Moreover, the size of the oscillations and the width of the inter-region are determined by the size of the averaging domain, and this is consistent with a previous analytical study performed in a simplified geometry. ${ }^{39}$ It must be noted that the oscillation, characterized by an average velocity value just below the porous medium-fluid boundary smaller than the average velocity in the homogeneous part of the porous medium, is a signature of pore-scale recirculations occurring in the very first layers of solid grains in the porous medium. These recirculations involve negative velocities in the $x$ direction, which, on average, contribute to significantly lower the macroscopic velocity (see, for instance, Figs. 4 and 5 in the work by Bruneau et al. ${ }^{8}$ for a similar behavior). Note also that this occurs regardless the flow regime (creeping or inertial). Finally, it must be emphasized that, in all the cases reported in this figure, there is an excellent agreement between the DNS results and the predictions from the ODA as the relative error percent between the solutions, taking the DNS results as the reference, is below $0.1 \%$.

\section{B. Inertial flow regime}

The second study case corresponds to inertial flow still under noslip conditions, considering the porous structure of Type 1 with a porosity equal to 0.8 and $r_{0}^{*}=1$. In contrast to the previous case, the height of the free fluid channel is decreased to be equal to only $5 \ell_{c}$. This choice is made to easily appreciate the entire velocity profiles in a single graph. In addition, this allows for the simulations to be less computationally demanding, as the presence of inertia requires much finer grids to reach convergence compared to the creeping flow regime.

The velocity profiles resulting from the ODA and the DNS are reported in Fig. 6, where, once again, excellent agreement is found. Simulations were performed for three values of the Reynolds number remaining smaller than the critical value corresponding to the first Hopf bifurcation. ${ }^{40}$ From these results, it is clear that the influence of inertia is experienced mostly in the homogeneous part of the porous medium, whereas its effects decrease as $z \rightarrow 0$. This observation is

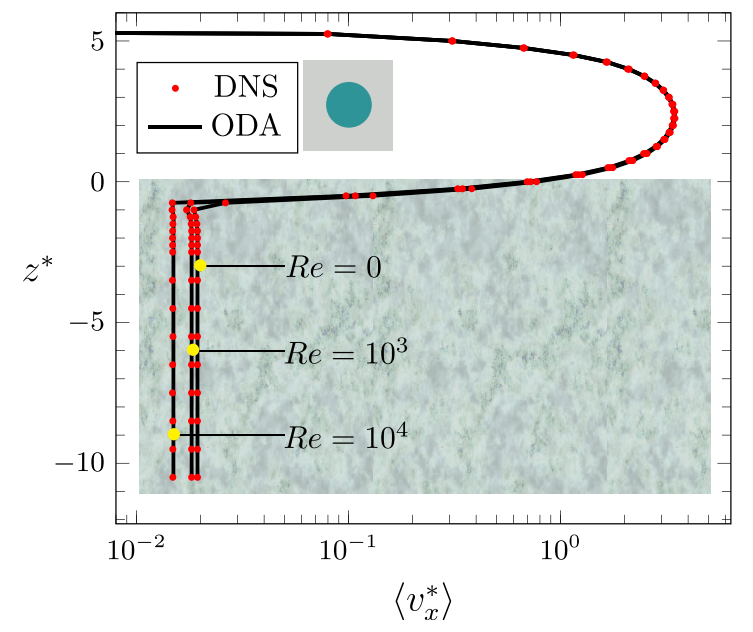

FIG. 6. Average velocity profiles for three Reynolds number values corresponding to a porosity value of 0.8 , fixing $r_{0}^{*}=1$ and $h^{*}=5$. Porous structure of Type 1 . 
consistent with the recent numerical results from Yang et al., ${ }^{14}$ which were obtained for squared-shape inclusions. This is due to the fact that the flow remains unidirectional and non-inertial in the free fluid region for these values of $R e$. Evidently, taking $z_{\omega}=11 \ell_{c}$ is an overestimate of the inter-region size and the same results are recovered using smaller values of $z_{\omega}$. As expected, the average velocity in the $\omega$-region decreases as $R e$ increases, and this is consistent with the fact that the apparent permeability is also decreasing when inertial effects are more intense. $^{33}$ These results serve to validate the ODA derived here under inertial conditions.

\section{Creeping slip-flow}

The final case study corresponds to slip flow under non-inertial conditions, i.e., $R e=0$. As in the previous case, the simulations are performed in a channel of height $h=5 \ell_{c}$, using the porous structure of Type 1 , with $\varepsilon=0.8$ and $r_{0}^{*}=1$. The average velocity profiles corresponding to the ODA and the DNS are reported in Fig. 7(a) for the situation in which the cell-Knudsen numbers in the porous medium and at the upper wall are equal, i.e., $\xi_{1} \lambda^{*}=\xi_{2} \lambda^{*}=\xi \lambda^{*}$. More specifically, four values of $\xi \lambda^{*}$ are examined, with the largest one remaining $\mathbf{O}(0.1)$ in order to avoid using the governing flow equations beyond their range of validity (i.e., out of the slip regime). ${ }^{41,42}$ The use of the same slip coefficient in both the porous medium and at the top wall of the free-fluid region is justified by the fact that the characteristic length of the latter region is not much larger than the one in the porous medium in the particular configuration considered here. Nevertheless, since the boundary condition given in Eq. (1c) may also be conceived as an effective boundary condition over rough surfaces, it is certainly possible to encounter physical situations in which $\xi_{1} \lambda^{*}=\xi_{2} \lambda^{*}$ when the contrast of the characteristic lengths is more pronounced.

Clearly, increasing the interfacial slip translates into an increase in the velocity profiles in the entire system, as expected. It is worth noting that, under no-slip conditions, there is a zone in the inter-region (near $z^{*}=-1$ ) where there is flow-reversal. This zone of flow reversal vanishes as slip effects become more important, i.e., for $\xi \lambda^{*}>0.1$.

It is also interesting to examine the influence of slip in the free fluid region. To this end, in Fig. 7(b), the velocity profiles corresponding to the case in which the cell Knudsen numbers are both equal to 0.5 are compared with those resulting from taking $\xi_{1} \lambda^{*}=0.5$ and $\xi_{2} \lambda^{*}=0$. The velocity profiles in the porous medium are the same in both situations; however, the maximum velocity values in the free fluid region are clearly decreased when the channel is assumed to be free of any rarefaction effects. Finally, it is important to mention that the predictions of the average velocity from the DNS and the ODA are in excellent agreement in all the physical situations considered here.

\section{Comparison with experimental data}

To conclude this section, it is pertinent to make one final validation of the macroscopic model by comparison with experiments. In specific, the experimental system studied by Terzis et al. ${ }^{5}$ is considered. This configuration is similar to the one used by Beavers and Joseph with the difference that the porous medium is made of an array of inline micropillars having a square cross section of $240 \mu \mathrm{m} \times 240 \mu \mathrm{m}$. The pillars measured $200 \mu \mathrm{m}$, which corresponds to the size of the system in the direction orthogonal to the plane of the mean flow. Since the pillars are placed $240 \mu \mathrm{m}$ apart from each other, the porosity is
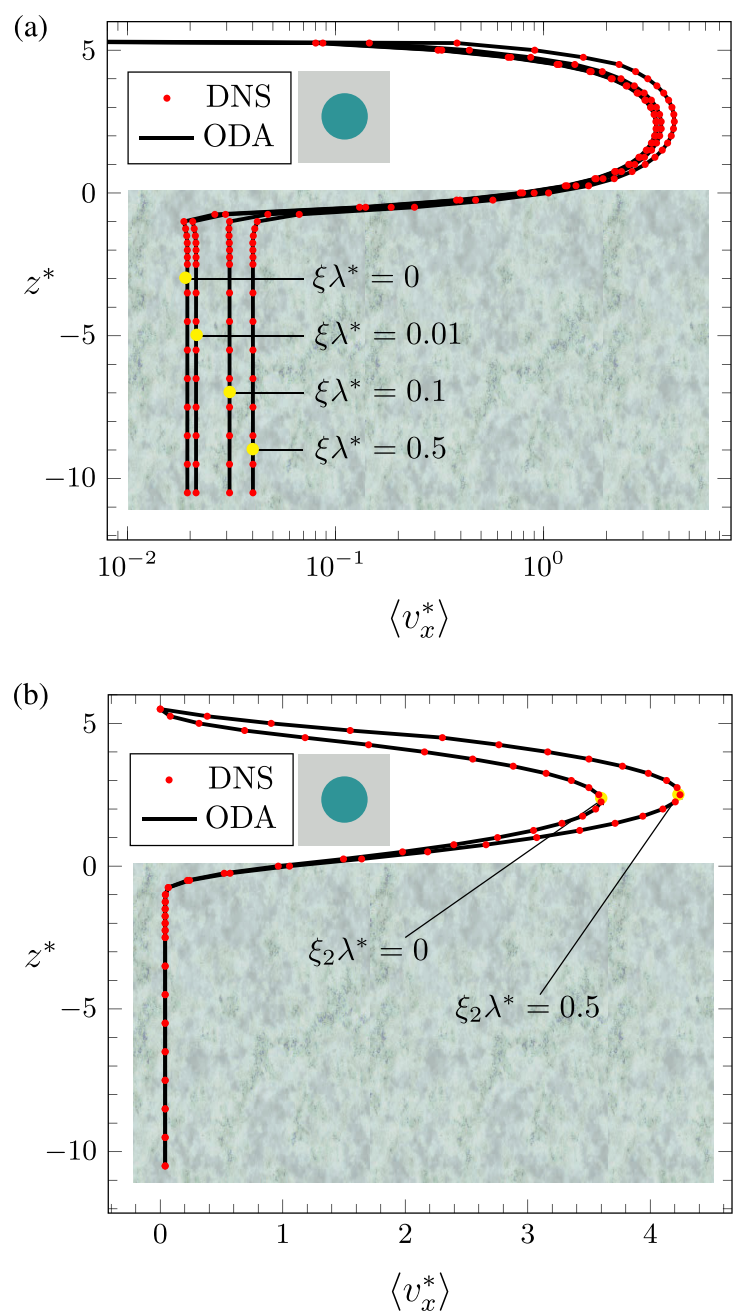

FIG. 7. Analysis of the slip effect on the average velocity profiles under creeping flow conditions for (a) $\xi_{1}=\xi_{2}=\xi$ and (b) $\xi_{1} \lambda^{*}=0.5$. Porous structure of Type 1 with $\varepsilon=0.8 . r_{0}^{*}=1$ and $h^{*}=5$.

equal to 0.75 . The height of the free-flow channel is $h=2 \mathrm{~mm}$, whereas the height of the porous medium region is $9.84 \mathrm{~mm}$. The flow conditions correspond to incompressible, steady, Newtonian, and creeping flow subject to no-slip at the solid-fluid interfaces. The experimental velocity measurements were performed using a micro PIV device. The velocity fields obtained with this instrument are line averages in the $y$-direction of the sketch shown in Fig. 1 (or equivalently, the $z$-direction in the work by Terzis et al.). In this way, the two-dimensional velocity fields shown in Fig. 4 of Terzis et al..$^{5}$ were obtained. These results were subsequently averaged along the $x$-direction in zones near the entrance, the outlet and in the middle of the system as shown in Fig. 9 of the above reference, yielding what is referred to as the cross-sectional average velocity, denoted $\left\langle v_{x}^{*}\right\rangle_{c s}$ in what follows. The experimental results taken at the middle of the system are now compared to the predictions of the model resulting from the ODA presented above. 
Due to the configuration of the experimental device, the closure problem solutions were carried out in a three-dimensional domain containing fifteen pillars in the vertical direction and one unit cell of the pillars pattern in the $x$-direction in order to be consistent with the results reported in Fig. 9 in Terzis et al..$^{5}$ It is worth noting that, in this case, the analytical solution derived above for the $\eta$-region is not applicable because the flow in this region is not one-dimensional. To address this issue, $z_{\eta}$ was set equal to $h$ so that the boundary condition given in Eq. (25a) was replaced by the following one:

$$
\mathbf{D}=\mathbf{0}, \quad \text { at } z=h .
$$

As a consequence, the $\eta$-region is no longer present in the model and is entirely lumped in the $\eta-\omega$ region. Once the fields of $\mathbf{D}$ and $\mathbf{F}$ were obtained, the pointwise velocity fields were reconstructed by making use of Eqs. (8a) and (24a), and they were further averaged in the plane-parallel to the macroscopic pressure gradient direction (i.e., the $x y$-plane) for several values of $z$. In this way, the spatial variations of the dimensionless cross-sectional average velocity, $\left\langle v_{x}^{*}\right\rangle_{c s}$, were predicted. Following Terzis et al., ${ }^{5}$ these results are made dimensionless by taking the magnitude of the inlet velocity of the system as $v_{r e f}$. The resulting velocity profiles are reported in Fig. 8(a), showing an excellent agreement with the experimental data, thus validating the ODA in this $3 \mathrm{D}$ flow situation. Notice that due to the shape of the experimental system, i.e., the wall effects in the $y$-direction, the velocity profiles tend to reach a constant value in the free fluid region sufficiently far away from the upper wall and the porous medium surface.

To complete the analysis of this system, it is worth studying the spatial variations of the superficial average of the velocity. The predictions of $\left\langle v_{x}^{*}\right\rangle$ resulting from both the ODA and the DNS are presented in Fig. 8(b), showing also an excellent agreement. The profiles exhibit a smooth behavior that contrasts with the experimental results from Terzis et al., ${ }^{5}$ which are included in this figure as a reference. Clearly, the values of $\left\langle v_{x}^{*}\right\rangle_{\text {cs }}$ oscillate around those of $\left\langle v_{x}^{*}\right\rangle$. Both types of averages are not expected to match since they correspond to different averaging domains.

\section{CONCLUSIONS}

In this work, a new formulation for describing steady, Newtonian, and incompressible flow between a porous medium and a fluid was derived. The model consists of a single Darcy-type equation that is written in terms of a position-dependent apparent permeability tensor. The values of this coefficient are predicted from the solution of the associated closure problems that are computationally much less demanding than performing direct numerical simulations. Although the model was formulated for the physical system configuration studied by Beavers and Joseph, it was derived in a somewhat general fashion in which flow may be multidimensional in the porous medium due to anisotropy, for instance. Moreover, the development was performed on the basis of the Navier-Stokes model at the porescale, allowing to account for inertial effects when the Reynolds number value justifies it. It also includes the potential existence of slip effects at the solid-fluid interfaces, which may occur when the cell-Knudsen number is not exceedingly small compared to unity, or when an effective slip boundary condition, resulting from roughness effects at the solid-fluid interfaces, is taken into account.

The predictive capabilities of the model were validated through in silico experiments by comparing the average velocity profiles resulting from the new ODA with pore-scale DNS, finding excellent agreement (i.e., with relative percent error values with respect to the DNS smaller than $0.1 \%$ ). The validation was carried out in three specific flow situations, namely: (1) creeping flow under no-slip conditions, (2) inertial flow also assuming no-slip conditions, and (3) creeping flow with slip. For flow under creeping and no-slip conditions, the effect of the porous medium microstructure was examined as well as the influence of the averaging domain size used to represent the flow in an average sense. The microstructure plays a key role in the magnitude and shape of the flow field not only in the homogeneous part of the porous medium, but also in the inter-region. The impact in the free-fluid region is significant in only a thin layer next to the porous medium-fluid boundary. Moreover, it was also shown that the
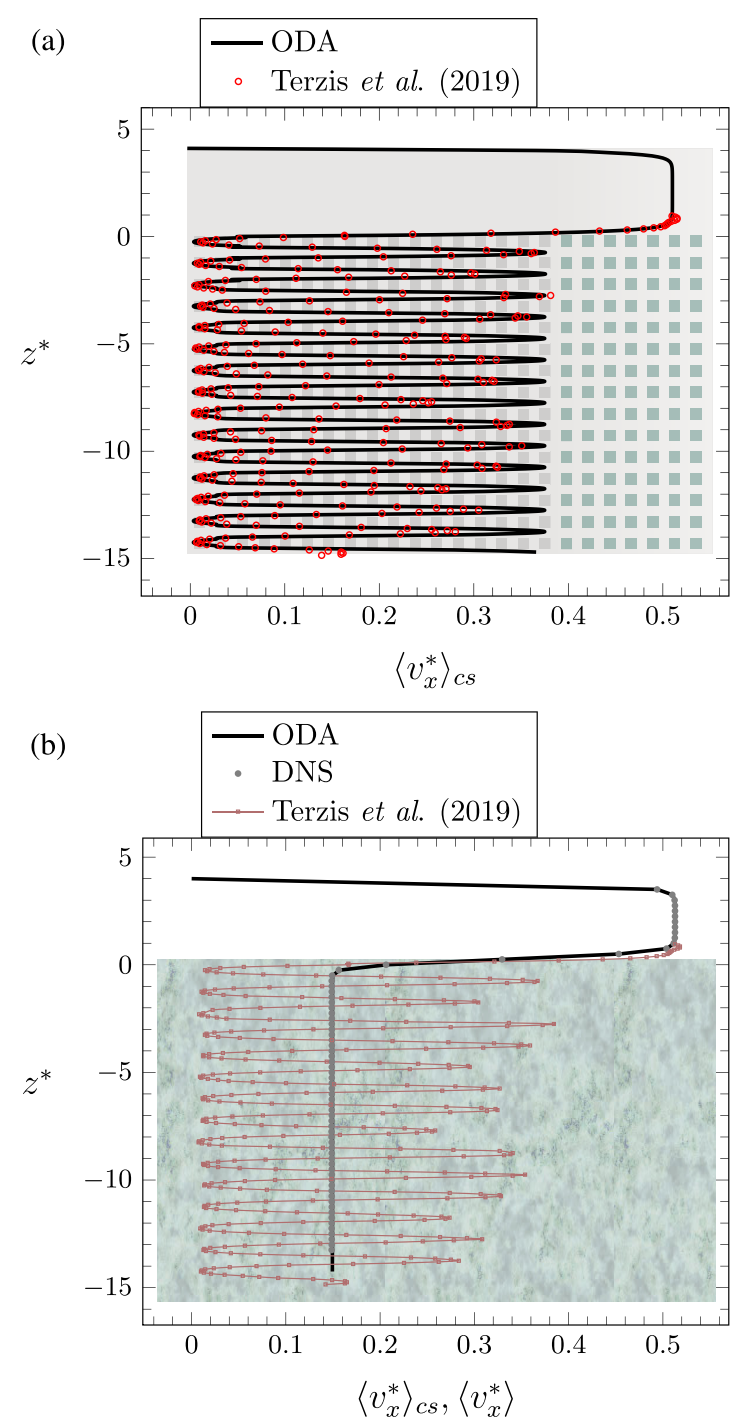

FIG. 8. (a) Comparison of the cross-sectional averaged velocity predictions from the ODA with the experimental data from Terzis et al. ${ }^{5}$ (b) Predictions of the superficial average velocity from the ODA and DNS. In all cases, the velocity is made dimensionless with respect to the inlet velocity. 
size of the inter-region is determined by the averaging domain size. For inertial and no-slip flow, the effects of changing the Reynolds number are mostly observed in the homogeneous porous medium and decrease near the fluid-porous medium boundary. Finally, the analysis in the creeping and slip-flow regime showed that slip effects play a crucial role in the velocity profiles in the entire system in the case in which slip effects are present in both the porous medium and the free-fluid regions. When the slip condition is imposed only in the porous medium, the velocity in the free fluid region experiences the most drastic changes.

In addition to the in silico validation, the ODA model was shown to reproduce the experimental results from Terzis et al., ${ }^{5}$ which were obtained under creeping flow conditions subject to no-slip at the solid-fluid interfaces. The results reported in Fig. 8 show that the cross-sectional averaged values of the velocity predicted from using the ODA reproduce adequately the available velocity profiles recorded in the middle of the micromodel device used by these authors. From the above, it is concluded that the new one-domain approach developed here is quite practical as it represents accurately data from both direct numerical simulations and experiments at a reasonable computational cost. Note that differently from previous attempts of formulating the ODA, it is not required to solve a PDE with positiondependent coefficients. Instead, a Darcy-like model is presented here that accounts for the spatial variations of the apparent permeability tensor in the inter-region that are given by the closure problem solution. This is also more practical than current versions of the twodomain approach where this information is required in an iterative process to compute the involved jump coefficients.

The results of this work should serve as a motivation to further study the one-domain approach and avoid regarding it as a model of archival value that is required in the definition of excess quantities in a two-domain approach. In addition, rarefaction effects beyond the slip regime and the use of effective boundary conditions on rough surfaces deserve to be studied in more detail by means of laboratory and direct numerical simulations in a wider context than the one envisaged here.

\section{ACKNOWLEDGMENTS}

The authors are thankful to A. Terzis for providing the experimental data used for the comparison with the model. D.L. is thankful to ANR for the support of Project No. ANR-11-RSNR0012-01, within which part of this work was carried out.

\section{DATA AVAILABILITY}

The data that support the findings of this study are available from the corresponding author upon reasonable request.

\section{REFERENCES}

'G. Beavers and D. Joseph, "Boundary conditions at a naturally permeable wall," J. Fluid Mech. 30, 197 (1967)

${ }^{2}$ I. Rybak, C. Schwarzmeier, E. Eggenweiler, and U. Rüde, "Validation and calibration of coupled porous-medium and free-flow problems using pore-scale resolved models," Comput. Geosci. (published online, 2020).

${ }^{3}$ A. Goharzadeh, A. Khalili, and B. B. Jørgensen, "Transition layer thickness at a fluid-porous interface,” Phys. Fluids 17, 057102 (2005).

${ }^{4}$ M. R. Morad and A. Khalili, "Transition layer thickness in a fluid-porous medium of multi-sized spherical beads," Exp. Fluids 46, 323-330 (2009).

${ }^{5}$ A. Terzis, I. Zarikos, K. Weishaupt, G. Yang, X. Chu, R. Helmig, and B. Weigand, "Microscopic velocity field measurements inside a regular porous medium adjacent to a low Reynolds number channel flow," Phys. Fluids 31, 042001 (2019).

${ }^{6}$ F. J. Valdés-Parada, J. Alvarez-Ramírez, B. Goyeau, and J. A. Ochoa-Tapia, "Computation of jump coefficients for momentum transfer between a porous medium and a fluid using a closed generalized transfer equation," Transp. Porous Media 78, 439-457 (2009).

${ }^{7}$ H. Chen and X.-P. Wang, "A one-domain approach for modeling and simulation of free fluid over a porous medium,” J. Comput. Phys. 259, 650-671 (2014).

${ }^{8}$ C.-H. Bruneau, D. Lasseux, and F. J. Valdés-Parada, "Comparison between direct numerical simulations and effective models for fluid-porous flows using penalization,” Meccanica 55, 1061-1077 (2020).

9 M. Sahraoui and M. Kaviany, "Slip and no-slip velocity boundary conditions at interface of porous, plain media,” Int. J. Heat Mass Transfer 35, 927-943 (1992).

${ }^{10}$ A. Nabovati and C. H. Amon, "Hydrodynamic boundary condition at openporous interface: A pore-level lattice Boltzmann study," Transp. Porous Media 96, 83-95 (2013).

${ }^{11} \mathrm{G}$. Neale and W. Nader, "Practical significance of Brinkman's extension of Darcy's law: Coupled parallel flows within a channel and a bounding porous medium," Can. J. Chem. Eng. 52, 475-478 (1974).

${ }^{12}$ J. Ochoa-Tapia and S. Whitaker, "Momentum transfer at the boundary between a porous medium and a homogeneous fluid-I. theoretical development," Int. J. Heat Mass Transfer 38, 2635-2646 (1995).

${ }^{13}$ F. J. Valdés-Parada, C. G. Aguilar-Madera, J. A. Ochoa-Tapia, and B. Goyeau, "Velocity and stress jump conditions between a porous medium and a fluid," Adv. Water Resources 62, 327-339 (2013).

${ }^{14}$ G. Yang, E. Coltman, K. Weishaupt, A. Terzis, R. Helmig, and B. Weigand, "On the Beavers-Joseph interface condition for non-parallel coupled channel flow over a porous structure at high Reynolds numbers," Transp. Porous Media 128, 431-457 (2019).

${ }^{15} \mathrm{E}$. Eggenweiler and I. Rybak, "Unsuitability of the Beavers and Joseph interface condition for filtration problems," J. Fluid Mech. 892, A10 (2020).

${ }^{16}$ D. A. Nield, "The Beavers-Joseph boundary condition and related matters: A historical and critical note," Transp. Porous Media 78, 537-540 (2009).

${ }^{17}$ J.-L. Auriault, "About the Beavers and Joseph boundary condition," Transp. Porous Media 83, 257-266 (2010).

${ }^{18}$ S. Whitaker, The Method of Volume Averaging (Springer, Netherlands, 1999).

${ }^{19}$ N. Luminari, G. A. Zampogna, C. Airiau, and A. Bottaro, "A penalization method to treat the interface between a free-fluid region and a fibrous porous medium," J. Porous Media 22, 1095-1107 (2019).

${ }^{20} \mathrm{~J}$. Chastanet, P. Royer, and J.-L. Auriault, "Does Klinkenberg's law survive upscaling?," Transp. Porous Media 56, 171-198 (2004).

${ }^{21}$ J. Chastanet, P. Royer, and J.-L. Auriault, "Flow of low pressure gas through dual-porosity media," Transp. Porous Media 66, 457-479 (2007).

${ }^{22}$ R. Venegas, C. Boutin, and O. Umnova, "Acoustics of multiscale sorptive porous materials,” Phys. Fluids 29, 082006 (2017).

${ }^{23}$ D. Einzel, P. Panzer, and M. Liu, "Boundary condition for fluid flow: Curved or rough surface,” Phys. Rev. Lett. 64, 2269-2272 (1990).

${ }^{24}$ R. Barber, Y. Sun, X. Gu, and D. Emerson, "Isothermal slip flow over curved surfaces," Vacuum 76, 73-81 (2004).

${ }^{25}$ E. Lauga, M. Brenner, and H. Stone, "Microfluidics: The no-slip boundary condition," in Springer Handbook of Experimental Fluid Mechanics (Springer, Berlin Heidelberg, 2007), pp. 1219-1240.

${ }^{26}$ D. Lasseux, F. J. V. Parada, J. A. O. Tapia, and B. Goyeau, "A macroscopic model for slightly compressible gas slip-flow in homogeneous porous media," Phys. Fluids 26, 053102 (2014).

${ }^{27}$ E. Skjetne and J.-L. Auriault, "Homogenization of wall-slip gas flow through porous media,” Transp. Porous Media 36, 293-306 (1999).

${ }^{28}$ J. Chastanet, P. Royer, and J.-L. Auriault, “Acoustics with wall-slip flow of gassaturated porous media,” Mech. Res. Commun. 31, 277-286 (2004).

${ }^{29}$ S. Pasquier, M. Quintard, and Y. Davit, "Modeling flow in porous media with rough surfaces: Effective slip boundary conditions and application to structured packings," Chem. Eng. Sci. 165, 131-146 (2017).

${ }^{30} \mathrm{G}$. Zampogna, J. Magnaudet, and A. Bottaro, "Generalized slip condition over rough surfaces,” J. Fluid Mech. 858, 407-436 (2019).

${ }^{31}$ F. A. Howes and S. Whitaker, "The spatial averaging theorem revisited," Chem. Eng. Sci. 40, 1387-1392 (1985). 
${ }^{32}$ S. Whitaker, “The Forchheimer equation: A theoretical development," Transp. Porous Media 25, 27-61 (1996).

${ }^{33}$ D. Lasseux, A. A. A. Arani, and A. Ahmadi, "On the stationary macroscopic inertial effects for one phase flow in ordered and disordered porous media," Phys. Fluids 23, 073103 (2011).

${ }^{34}$ D. Lasseux, F. J. V. Parada, and M. L. Porter, "An improved macroscale model for gas slip flow in porous media,” J. Fluid Mech. 805, 118-146 (2016).

${ }^{35}$ J. Barrere, O. Gipouloux, and S. Whitaker, "On the closure problem for Darcy's law," Transp. Porous Media 7, 209-222 (1992).

${ }^{36}$ W. G. Gray, "A derivation of the equations for multi-phase transport," Chem. Eng. Sci. 30, 229-233 (1975).

${ }^{37}$ D. Lasseux and F. J. Valdés-Parada, "Symmetry properties of macroscopic transport coefficients in porous media,” Phys. Fluids 29, 043303 (2017).
${ }^{38}$ Z. Chai, J. Lu, B. Shi, and Z. Guo, "Gas slippage effect on the permeability of circular cylinders in a square array,” Int. J. Heat Mass Transfer 54, 3009-3014 (2011).

${ }^{39}$ J. Ochoa-Tapia, F. Valdés-Parada, B. Goyeau, and D. Lasseux, "Fluid motion in the fluid/porous medium inter-region,” Rev. Mex. Ing. Quím. 16, 923-938 (2017).

${ }^{40} \mathrm{M}$. Agnaou, D. Lasseux, and A. Ahmadi, "From steady to unsteady laminar flow in model porous structures: An investigation of the first Hopf bifurcation," Comput. Fluids 136, 67-82 (2016).

${ }^{41}$ V. F. Kozlov, A. V. Fedorov, and N. D. Malmuth, "Acoustic properties of rarefied gases inside pores of simple geometries," J. Acoust. Soc. Am. 117, 3402-3411 (2005).

${ }^{42}$ G. Karniadakis, A. Beskok, and N. Aluru, Microflows and Nanoflows (Springer, New York, 2005) 